\title{
Effect of Mineral Nitrogen and Legume Intercrops on Maize (Zea Mays L.) Nitrogen Uptake, Nutrient Use Efficiency and Yields in Chitedze and Zomba, Malawi
}

\author{
Innocent Malunga ${ }^{1}$, Joyce J. Lelei ${ }^{1} \&$ Wilkson Makumba ${ }^{2}$ \\ ${ }^{1}$ Department of Crops, Horticulture and Soils, Egerton University, P.O. Box 536 20115, Egerton, Njoro Kenya \\ ${ }^{2}$ Department of Agricultural Research Services (DARS), P.O. Box 30779, Lilongwe 3, Malawi \\ Correspondence: Innocent Malunga, Department of Crops, Horticulture and Soils, Egerton University, P.O. Box \\ 536 20115, Egerton, Njoro Kenya. Tel: 265-419-932/ 254-790-406-905. E-mail: innocentmalunga@ gmail.com
}

\author{
Received: September 27, 2017 Accepted: October 11, 2017 Online Published: October 31, 2017 \\ doi:10.5539/sar.v7n1p64 \\ URL: https://doi.org/10.5539/sar.v7n1p64
}

\begin{abstract}
Nitrogen $(\mathrm{N})$ is the most critical plant nutrient. Maize has a high nitrogen demand. Low maize (Zea mays L.), yields in smallholder farms of Malawi, the country's staple, is attributable to declining $\mathrm{N}$ fertility aggravated by the ever increasing price of fertilizer. Little effort has been made to establish the best nitrogen rate in a maizecowpea and maize-bean intercrop under variable soil conditions as a way of improving production, and was the objective of the current study. Field experiments were conducted at Chitedze Agricultural Research Station in Lilongwe and Makoka Agricultural Research Station in Zomba during the 2016/17 growing season. A split plot layout in a randomized complete block design, with three replicates was used. The main plots were; sole maize, sole bean, sole cowpea, bean/maize and cowpea/maize intercrop systems. The sub plots were $\mathrm{N}$ fertilizer rates $(0$, 52.5, 78.75 and $105 \mathrm{~kg} \mathrm{~N} \mathrm{ha}^{-1}$ ), applied as urea. The data collected was subjected to analysis of variance using SAS software version 9.3 (SAS Institute Inc.) at $\mathrm{P}<0.05$. Means were separated using Duncan Multiple Range (DMRT) test at $95 \%$ significance level. The results showed that application of $\mathrm{N}$ increased maize $\mathrm{N}$ uptake and grain yield. NUE however decreased with increasing $\mathrm{N}$ fertilizer rate.
\end{abstract}

Keywords: common beans, cowpea, urea

\section{Introduction}

Nitrogen $(\mathrm{N})$ is a critical component of organic molecules such as amino acids, proteins and nucleic acids (Walworth, 2013) and promotes vigorous vegetative growth (Havlin, Tisdale, Beaton, \& Werner, 2008; Paul, 2008). It is the most frequently deficient plant nutrient in Malawian soils. This has resulted into low maize (Zea mays L.) yields, the country's staple (Munthali \& Mazuma, 2010). The deficiency of $\mathrm{N}$ in soil is as a result of continuous cropping without adequate replenishment of nutrients lost through harvesting. Nitrogen $(\mathrm{N})$ is one of the essential nutrients affected by increased soil cropping (Gachene \& Kimaru, 2003; Mbewe, 2011). Alternative ways of improving nitrogen fertility of soil and subsequently increase maize yield should be identified.

Studies conducted on legumes have reported that legumes have the potential to significantly contribute to soil nitrogen, through biological nitrogen fixation, and increase yields of either subsequent or associated non-nodulating crops such as cereals through biological nitrogen fixation (Hayat, Safdar, Siddique, \& Chatha, 2008).

Cowpea (Vigna unguiculata L) is a legume of African origin and one of the most ancient crops ever domesticated by man. It is an important source of food and income (Food and Agriculture Organisation [FAO], 2004). Africa produces $96 \%$ of the more than 5.4 million tons of the global cowpea production. Cowpea is highly adaptable to grow in different soil types and intercropping systems. It also has the following advantages; it is drought resistant and has the ability to improve soil fertility through biological nitrogen fixation and also reduces the risk of soil erosion (International Institute of Tropical Agriculture [IITA], 2016). In Malawi cowpea is an important legume crop for small holder farmers and the crop is adapted to grow in a wide range of local conditions (Nkongolo, Bokosi, Malusi, Vokhiwa, \& Mphepo, 2009). The production of cowpea in Malawi is estimated to be 50249 million tons on average between the years 2000 to 2013 (FAOSTAT, 2013). 
Common bean (Phaseolus vulgaris $\mathrm{L}$ ) is the most important food legume for direct consumption in the world and it is a major source of dietary protein in Malawi. It is also an important cash crop for smallholder farmers in Malawi (FAO, 1999; Magreta \& Jambo, 2012). International Crops Research Institute for the Semi-Arid Tropics [ICRISAT] (2013) estimated the production of beans in Malawi to be about 111889 million tones on average between 2000- 2011. There is a gap in information on the best nitrogen rate in a maize- cowpea and maize-bean intercrop under variable soil conditions as a way of improving production.

The current study investigated the effect of legume intercrops and mineral nitrogen on nutrient uptake, nutrient use efficiency and grain yield of maize (Zea mays L.) in Malawi.

\section{Method}

2.1 Site

Field experiments were conducted in two sites in 2016/17 growing season. The first site was the Chitedze Agricultural Research Station, near Lilongwe in the Central part of Malawi. It is located at about $13^{\circ} 59^{\circ} \mathrm{S}$ longitude and $33^{\circ} 38^{\circ} \mathrm{E}$ latitude at an elevation of $1146 \mathrm{~m}$ asl. Chitedze research station has a mean annual temperature of $20^{\circ} \mathrm{C}$ and receives an average annual rainfall of 800 to $900 \mathrm{~mm}, 85 \%$ of which falls from November to April. The station is a representative of Lilongwe plain, which is a major maize producing agro-ecology of the country. The terrain is flat to gently undulating. The soils are chromic Luvisols. They have a well-developed structure with a dark, reddish brown top soil. The $\mathrm{pH}$ ranges from 4.5 to 6.0.

The second site was Makoka Agricultural Research Station near Zomba, in the southern region of Malawi. The soil is classified as a Ferric Lixisol (FAO/UNESCO) or Oxic Hapleustalf (USDA). It is situated at about $15^{\circ} 30^{\prime} \mathrm{S}$ longitude and $35^{\circ} 15^{\prime} \mathrm{E}$ latitude at an elevation of $1030 \mathrm{~m}$ above sea level. The soil texture is $46 \%$ sand, $46 \%$ clay and $8 \%$ silt. Total annual rainfall ranges from 560 to $1600 \mathrm{~mm}$, with a mean of $1024 \mathrm{~mm}$. The site experiences unimodal type of rainfall with most of the rains falling from November to April.

Chemical and physical characteristics of soils at Chitedze and Makoka Agricultural research stations are shown in Table 1.

Table 1. Initial physical and chemical characteristics of soils at Chitedze and Makoka Agricultural Research Stations

\begin{tabular}{llllll}
\hline & & Chitedze & & Makoka & \\
\hline Property & Units & Value & Interpretation* & Value & Interpretation* \\
\hline PH & - & 6.09 & Medium & 4.91 & Deficient in Ca \\
Organic Carbon & $\%$ & 1.58 & Medium & 0.32 & Low \\
Organic Matter & $\%$ & 2.75 & Adequate & 0.53 & Low \\
Total N & $\%$ & 0.20 & Medium & 0.10 & Low \\
Available P & $\mathrm{ppm}$ & 39.54 & High & 72.98 & High \\
$\mathrm{K}$ & $\mathrm{cmol}_{\mathrm{c} \mathrm{kg}} \mathrm{kg}^{-1}$ & 0.11 & Low & 0.11 & Low \\
$\mathrm{Ca}$ & $\mathrm{cmol}_{\mathrm{c} \mathrm{k}} \mathrm{kg}^{-1}$ & 0.45 & Low & 0.16 & Low \\
$\mathrm{Mg}$ & $\mathrm{cmol}_{\mathrm{c}} \mathrm{kg}^{-1}$ & 0.05 & Low & 0.04 & Low \\
$\mathrm{Zn}$ & $\mathrm{ppm}$ & 0.31 & Low & 0.10 & Low \\
Clay & $\%$ & 19.20 & & 19.33 & \\
Silt & $\%$ & 13.00 & & 6.83 & \\
Sand & $\%$ & 67.80 & & 73.84 & \\
Textural class & & Sandy clay loam & & Sandy clay loam & \\
\hline
\end{tabular}

*Chemical characteristics of soil were classified according to Landon (1991)

\subsection{Treatments and Experimental Design}

A split plot layout in a randomized complete block design (RCBD), with three replications, was used. The main plots were; cropping systems (sole maize, sole bean, sole cowpea, bean/maize and cowpea/maize intercrops). The sub plots were four rates of $\mathrm{N}\left(0,52.5,78.75\right.$ and $\left.105 \mathrm{~kg} \mathrm{~N} \mathrm{ha}^{-1}\right)$, applied as urea fertilizer. The inorganic fertilizer rates correspond to zero, one-half (50\%), $75 \%$ and $100 \%$ of the recommended national fertilizer rate for maize in Malawi. The recommended rate for Malawi is $105 \mathrm{~kg} \mathrm{~N} \mathrm{ha}^{-1}$ by side dressing (MAIFS, 2004). The sub plot sizes measured $1 \mathrm{~m} \times 7.5 \mathrm{~m}$. Space for foot path $(0.5 \mathrm{~m})$ between plots and blocks $(1 \mathrm{~m})$, was provided.

\subsection{Land Preparation and Planting}

Land was prepared manually using hand implements. Maize was planted at spacing of $75 \mathrm{~cm}$ between rows and 
$25 \mathrm{~cm}$ between planting stations. Two seeds were planted per hill and later thinned to 1 seedling, when plants were $10 \mathrm{~cm}$ tall $(53,000$ plants/ha). The maize variety SC 403 was planted. In the intercropping system, the legume seeds were sown between two maize rows, at spacing of $75 \mathrm{~cm}$ between rows and $25 \mathrm{~cm}$ between planting stations. Two seeds were planted per station, and then thinned to one plant. A medium duration common bean variety (Mwaiwathu alimi) was planted. The cowpea variety planted was IT82E-16. Sole legumes were sown at a spacing of $75 \mathrm{~cm}$ between rows and $25 \mathrm{~cm}$ between planting stations.

\subsection{Fertilizer Application and Field Management}

Urea fertilizer was applied as a source of nitrogen, in two equal splits; 7 and 30 days after planting, to minimize potential leaching losses. Triple superphosphate (TSP) was applied at a rate of $40 \mathrm{~kg} \mathrm{P} \mathrm{ha}^{-1}$ as basal dressing fertilizer. Careful and superficial manual weeding was done three times, after crop emergence. Pesticides (Dimethoate and Cypermethrin) were applied twice. The first application was done when the plants were two weeks old and the second when the maize was tasseling and the legumes were flowering. The pesticides were applied at a rate of 3litres/hectare.

\subsection{Soil and Plant Sampling}

Soil samples were collected using Edelman soil augers $(0-30 \mathrm{~cm})$ from experimental fields using traverse method, before application of treatments, and then composited. Thereafter top soil samples $(0-30 \mathrm{~cm})$ were collected at harvest of maize, randomly from three locations in each plot between planting stations within a row, and composited.

Maize plant samples were collected at $50 \%$ tasseling stage. The leaf opposite the ear was taken from five plants per plot. The plants were selected randomly from the plot border rows. At physiological maturity the above ground portion of the maize was harvested from two internal rows. Maize samples were divided into stover (stalk and leaves), cob and grains. For legumes at harvest, samples were collected from two internal rows and divided into stover and pods.

\subsection{Soil and Plant Analysis}

Soil samples were air dried to constant weight, for at least 96 hours, ground to pass through a $2 \mathrm{~mm}$ sieve and analyzed for organic $\mathrm{N}, \mathrm{C}$, extractable $\mathrm{P}$ and exchangeable $\mathrm{Ca}, \mathrm{Mg}$ and $\mathrm{K}$, following standard procedures stipulated by Anderson and Ingram (1993). Exchangeable cations were determined by using $1 \mathrm{~N}$ ammonium acetate. Soil $\mathrm{pH}$ was read in a suspension of 1:2.5 soils: distilled water. Texture was determined using a hydrometer in a dispersant solution of 3\% sodium hexametaphosphate (Anderson \& Ingram, 1993).

Plant samples collected (maize and legumes) were weighed and chopped into small pieces. Sub-samples oven dried at $65^{\circ} \mathrm{C}$ for 72 hours. The weights of the oven dry sub-samples were recorded. The dried maize samples were ground and digested in sulphuric acid- selenium extractant and analyzed for total nitrogen according to standard procedures stipulated by Anderson and Ingram (1993).

\subsection{Calculation Procedures}

Maize nutrient uptake: was calculated from nutrient concentrations and dry matter measured using the following formulae (Peterburgski, 1986);

$$
\text { Total nutrient uptake }=\text { nutrient } \text { concentration } \mathrm{x} \text { dry matter yield }
$$

Maize and legume Yield: Grain yield was expressed at $13 \%$ moisture content, determined by moisture meter. The weight of grains and dry matter were measured by an analytical balance and yield converted into $\mathrm{kg} \mathrm{ha}^{-1}$ using the following formulae:

$$
\text { Yield }(\mathrm{kg} / \mathrm{ha})=\text { yield }(\mathrm{kg}) / \mathrm{m}^{2}(\mathrm{~kg}) \times 10000
$$

Nutrient use efficiency: using treatment yield (NUE) was calculated using the following formula (Brentrup \& Palliere, 2010):

$$
\text { NUE }=\text { yield with nitrogen- yield without nitrogen/ total nitrogen added }
$$

\subsection{Statistical Analysis}

Data was subjected to analysis of variance (ANOVA) conducted using statistical package SAS version 9.3. The treatment means were separated using Duncan's Multiple Range Test (DMRT) tests at $\mathrm{P}<0.05$.

\section{Results}

\subsection{Effect of Cropping System, Nitrogen Level, Location and Their Interaction on Maize Grain Yield}

The main effects of nitrogen levels and locations on maize grain yield were significant (Table 2). Significantly 
higher values $(\mathrm{P}<0.05)$ were obtained with application of $105 \mathrm{~kg} \mathrm{~N} \mathrm{ha}^{-1}(\mathrm{~N} 3)$, followed by $78.75 \mathrm{~kg} \mathrm{~N} \mathrm{ha}^{-1}$ ( $\left.\mathrm{N} 2\right)$, $\mathrm{N} 1\left(52.5 \mathrm{~kg} \mathrm{~N} \mathrm{ha}^{-1}\right)$ and $\mathrm{N} 0\left(0 \mathrm{~kg} \mathrm{~N} \mathrm{ha}^{-1}\right)$, in that order. Maize grain yield was significantly higher at Chitedze than at Makoka. Effect of cropping system was not significant for maize grain yield .The effect of interaction of cropping systems $x$ levels of nitrogen was not significant for maize grain yield (Table 3 ). The nitrogen levels $x$ cropping systems $x$ location interactions effects were not significant $(\mathrm{P}<0.05)$ for maize yield grain yield (Table 4). The interactive effects of cropping system $\mathrm{x}$ location and nitrogen levels $\mathrm{x}$ location maize grain yield were not significant (Table 5).

Table 2. Effect of nitrogen level, cropping system and location on maize grain yield, DM yield and weight of cobs

\begin{tabular}{ll}
\hline Treatment & Maize grain Yield(Mt/ha) \\
\hline Nitrogen Level & \\
N3 & $6.25 \mathrm{a}$ \\
$\mathrm{N} 2$ & $5.37 \mathrm{~b}$ \\
$\mathrm{~N} 1$ & $3.99 \mathrm{c}$ \\
$\mathrm{N} 0$ & $1.54 \mathrm{~d}$ \\
Cropping system & \\
Maize- monocropping & $4.47 \mathrm{a}$ \\
bean/maize- intercropping & $4.16 \mathrm{a}$ \\
cowpea/maize- intercropping & $4.23 \mathrm{a}$ \\
Location & \\
Chitedze & $5.04 \mathrm{a}$ \\
Makoka & $3.54 \mathrm{~b}$ \\
\hline
\end{tabular}

Means with the same letter are not significantly different $(\mathrm{P}<0.05)$ according to Duncan test

Key: N0 $=0 \mathrm{~kg} \mathrm{~N}^{-1}, \mathrm{~N} 1=52.5 \mathrm{~kg} \mathrm{~N} \mathrm{ha}^{-1}, \mathrm{~N} 2=78.75 \mathrm{~kg} \mathrm{~N} \mathrm{ha}^{-1}, \mathrm{~N} 3=105 \mathrm{~kg} \mathrm{~N} \mathrm{ha}^{-1}$

Table 3. Effect of the interaction between cropping systems and levels of nitrogen on maize grain yield (means \pm std. error)

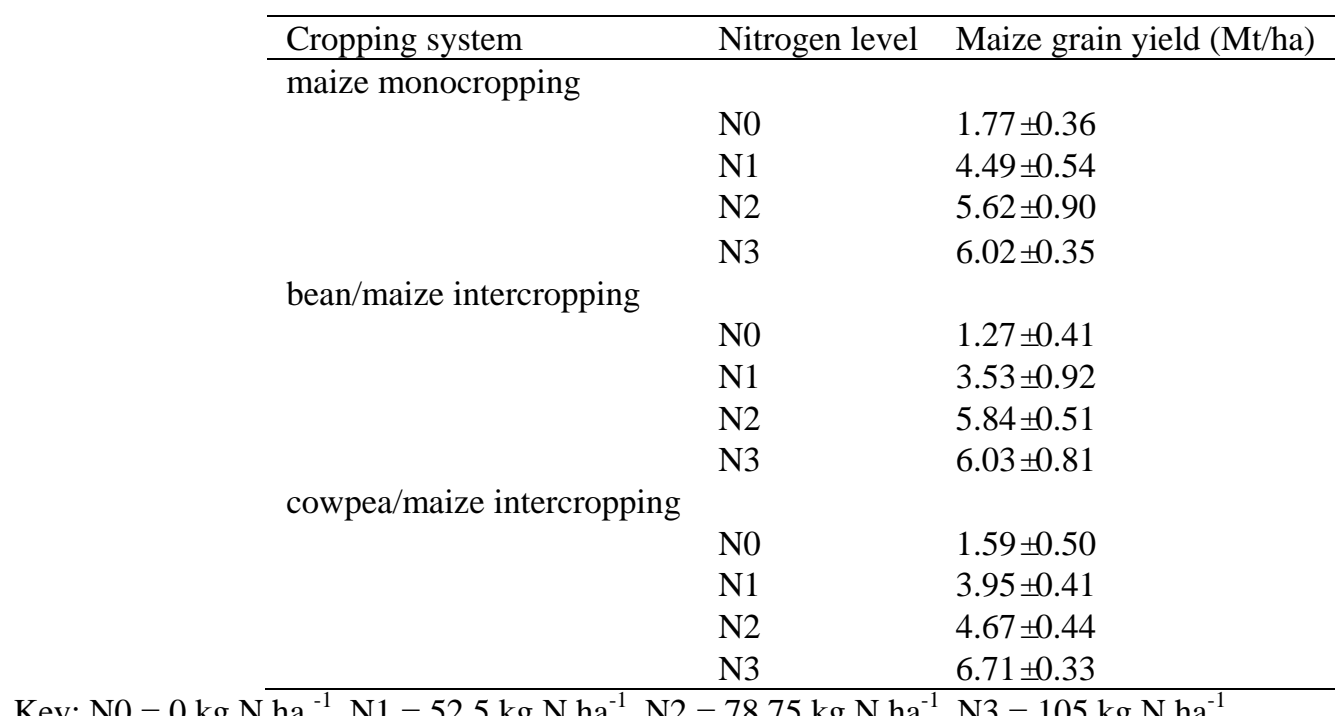


Table 4. Effect of interaction between cropping system $\mathrm{x}$ nitrogen level $\mathrm{x}$ location on maize yield (means \pm std. error)

\begin{tabular}{|c|c|c|c|}
\hline Cropping system & Nitrogen level & Location & Maize Yield (Mt/ha) \\
\hline \multirow[t]{9}{*}{ maize monocropping } & & & \\
\hline & \multirow[t]{2}{*}{ No } & Chitedze & $2.24 \pm 0.48$ \\
\hline & & Makoka & $1.30 \pm 0.43$ \\
\hline & \multirow[t]{2}{*}{ N1 } & Chitedze & $5.41 \pm 0.61$ \\
\hline & & Makoka & $3.57 \pm 0.46$ \\
\hline & \multirow[t]{2}{*}{$\mathrm{N} 2$} & Chitedze & $7.10 \pm 1.00$ \\
\hline & & Makoka & $4.14 \pm 0.95$ \\
\hline & \multirow[t]{2}{*}{ N3 } & Chitedze & $6.03 \pm 0.77$ \\
\hline & & Makoka & $6.00 \pm 0.14$ \\
\hline \multirow[t]{9}{*}{ bean/maize intercropping } & & & \\
\hline & \multirow[t]{2}{*}{ No } & Chitedze & $2.01 \pm 0.35$ \\
\hline & & Makoka & $0.52 \pm 0.42$ \\
\hline & \multirow[t]{2}{*}{ N1 } & Chitedze & $4.97 \pm 1.37$ \\
\hline & & Makoka & $2.10 \pm 0.55$ \\
\hline & \multirow[t]{2}{*}{$\mathrm{N} 2$} & Chitedze & $6.90 \pm 0.12$ \\
\hline & & Makoka & $4.77 \pm 0.40$ \\
\hline & \multirow[t]{2}{*}{ N3 } & Chitedze & $7.03 \pm 0.93$ \\
\hline & & Makoka & $5.03 \pm 1.18$ \\
\hline \multirow[t]{9}{*}{ cowpea/maize intercropping } & & & \\
\hline & \multirow[t]{2}{*}{ No } & Chitedze & $1.82 \pm 0.71$ \\
\hline & & Makoka & $1.35 \pm 0.85$ \\
\hline & \multirow[t]{2}{*}{ N1 } & Chitedze & $4.44 \pm 0.70$ \\
\hline & & Makoka & $3.46 \pm 0.31$ \\
\hline & \multirow[t]{2}{*}{$\mathrm{N} 2$} & Chitedze & $5.17 \pm 0.83$ \\
\hline & & Makoka & $4.16 \pm 0.21$ \\
\hline & \multirow[t]{2}{*}{ N3 } & Chitedze & $7.38 \pm 0.25$ \\
\hline & & Makoka & $6.05 \pm 0.13$ \\
\hline
\end{tabular}

Key N0 $=0 \mathrm{~kg} \mathrm{~N} \mathrm{ha}^{-1}, \mathrm{~N} 1=52.5 \mathrm{~kg} \mathrm{~N} \mathrm{ha}^{-1}, \mathrm{~N} 2=78.75 \mathrm{~kg} \mathrm{~N}^{-1}, \mathrm{~N} 3=105 \mathrm{~kg} \mathrm{~N} \mathrm{ha}^{-1}$.

Table 5. Effect of the interactions between cropping systems and location, nitrogen levels and location on maize yield (means \pm std. error)

\begin{tabular}{|c|c|c|}
\hline Treatment & Location & Maize grain yield (Mt/ha) \\
\hline \multicolumn{3}{|c|}{$\begin{array}{l}\text { Cropping system } \\
\text { maize monocropping }\end{array}$} \\
\hline & Chitedze & $5.19 \pm 0.63$ \\
\hline & Makoka & $3.75 \pm 0.56$ \\
\hline \multicolumn{3}{|c|}{ bean/maize intercropping } \\
\hline & Chitedze & $5.23 \pm 0.71$ \\
\hline & Makoka & $3.11 \pm 0.64$ \\
\hline \multicolumn{3}{|c|}{ cowpea/maize intercropping } \\
\hline & Chitedze & $4.71 \pm 0.66$ \\
\hline & Makoka & $3.75 \pm 0.54$ \\
\hline \multicolumn{3}{|c|}{ Nitrogen level } \\
\hline \multirow[t]{2}{*}{ N0 } & Chitedze & $2.02 \pm 0.27$ \\
\hline & Makoka & $1.06 \pm 0.33$ \\
\hline \multirow[t]{2}{*}{$\mathrm{N} 1$} & Chitedze & $4.94 \pm 0.50$ \\
\hline & Makoka & $3.04 \pm 0.33$ \\
\hline \multirow[t]{2}{*}{$\mathrm{N} 2$} & Chitedze & $6.39 \pm 0.48$ \\
\hline & Makoka & $4.35 \pm 0.32$ \\
\hline \multirow[t]{2}{*}{ N3 } & Chitedze & $6.82 \pm 0.41$ \\
\hline & Makoka & $5.69 \pm 0.38$ \\
\hline
\end{tabular}




\subsection{Effect of Cropping System, Nitrogen Level, Location and Their Interaction on Nitrogen Uptake by Maize}

There were significant $(\mathrm{P}<0.05)$ main effects of nitrogen levels and location on $\mathrm{N}$ uptake by maize. The highest uptake was observed in N3 $\left(105 \mathrm{~kg} \mathrm{~N} \mathrm{ha}^{-1)}\right.$ nitrogen level, followed by $\mathrm{N} 2\left(78.75 \mathrm{~kg} \mathrm{~N} \mathrm{ha}^{-1)}\right.$. The lowest amounts of nutrient uptake were recorded in N0 $\left(0 \mathrm{~kg} \mathrm{~N} \mathrm{ha}^{-1}\right)$ and N1 $\left(52.5 \mathrm{~kg} \mathrm{~N} \mathrm{ha}^{-1}\right)$ nitrogen levels. Significantly higher uptake was observed at Chitedze than Makoka. Cropping systems did not have any significant effect on nutrient uptake (Table 6). The interactive effect of cropping systems $\mathrm{x}$ nitrogen levels on $\mathrm{N}$ uptake by maize was significant $(\mathrm{P}<0.05)$ (Table 7). The uptake was significantly higher in bean/maize intercropping system followed by cowpea/maize intercropping under $\mathrm{N} 3$ level of nitrogen in both cropping systems and in maize monocropping when the nitrogen levels were $\mathrm{N} 1$ and $\mathrm{N} 2$.

The effect of cropping systems $\mathrm{x}$ location interaction on $\mathrm{N}$ uptake in maize was significant only under maize monocropping system. The uptake was significantly higher at Chitedze than at Makoka in maize monocropping system at both sites (Table 8). The interaction of nitrogen levels $\mathrm{x}$ location on $\mathrm{N}$ uptake by maize was not significant $(\mathrm{P}<0.05)$.The interaction effects of nitrogen level $\mathrm{x}$ cropping system $\mathrm{x}$ location on $\mathrm{N}$ uptake by maize was significant $(\mathrm{P}<0.05)$ (Table 9). Uptake was significantly higher under maize monocropping system combined with N3 level of nitrogen at Chitedze. The values were significantly lower under cowpea/maize intercropping system combined with N0 level of nitrogen at Makoka.

Table 6. Effects of cropping systems, nitrogen levels and locations on nutrient uptake in maize

\begin{tabular}{lc}
\hline Treatments & Nutrient uptake \\
\hline & (mg-N/plant) \\
\hline Cropping system & \\
maize monocropping & $132.13 \mathrm{a}$ \\
bean/maize intercropping & $117.92 \mathrm{a}$ \\
cowpea/maize intercropping & $122.68 \mathrm{a}$ \\
Nitrogen level & \\
N0 & $56.05 \mathrm{c}$ \\
N1 & $103.36 \mathrm{bc}$ \\
N2 & $139.41 \mathrm{~b}$ \\
N3 & $198.15 \mathrm{a}$ \\
Location & \\
Chitedze & $163.03 \mathrm{a}$ \\
Makoka & $85.45 \mathrm{~b}$ \\
\hline
\end{tabular}

Means with the same letter within a column are not significantly different $(\mathrm{P}<0.05)$ according to Duncan test Key: $\mathrm{N} 0=0 \mathrm{~kg} \mathrm{~N} \mathrm{ha}^{-1}, \mathrm{~N} 1=52.5 \mathrm{~kg} \mathrm{~N} \mathrm{ha}^{-1}, \mathrm{~N} 2=78.75 \mathrm{~kg} \mathrm{~N} \mathrm{ha}^{-1}, \mathrm{~N} 3=105 \mathrm{~kg} \mathrm{~N} \mathrm{ha}^{-1}$.

Table 7. Effect of interaction between nitrogen levels $\mathrm{x}$ cropping system on nutrient uptake in maize (means \pm std. error)

\begin{tabular}{lll}
\hline Cropping system & Nitrogen level & Nutrient uptake (mg-N/plant) \\
\hline maize monocropping & N0 & $53.07 \pm 10.53$ \\
& N1 & $123.49 \pm 25.02$ \\
& N2 & $132.39 \pm 16.65$ \\
bean/maize intercropping & N3 & $219.57 \pm 38.86$ \\
& N0 & $49.66 \pm 16.30$ \\
& N1 & $79.94 \pm 19.24$ \\
& N2 & $154.56 \pm 49.26$ \\
cowpea/maize intercropping & N3 & $187.49 \pm 44.08$ \\
& N0 & $65.42 \pm 25.32$ \\
& N1 & $106.65 \pm 41.92$ \\
& N2 & $131.28 \pm 39.25$ \\
& N3 & $187.38 \pm 55.81$ \\
\hline
\end{tabular}

Key: $\mathrm{N} 0=0 \mathrm{~kg} \mathrm{~N} \mathrm{ha}{ }^{-1}, \mathrm{~N} 1=52.5 \mathrm{~kg} \mathrm{~N} h a^{-1}, \mathrm{~N} 2=78.75 \mathrm{~kg} \mathrm{~N} \mathrm{ha}^{-1}, \mathrm{~N} 3=105 \mathrm{~kg} \mathrm{~N} \mathrm{ha}^{-1}$. 
Table 8. Effects of cropping system $\mathrm{x}$ location, nitrogen levels $\mathrm{x}$ location on nutrient uptake in maize (means \pm std. error)

\begin{tabular}{llc}
\hline Treatments & Location & Nutrient uptake (mg N/plant) \\
\hline Cropping System & & \\
maize monocropping & Chitedze & $151.85 \pm 28.00$ \\
& Makoka & $112.41 \pm 18.82$ \\
bean/maize intercropping & Chitedze & $156.23 \pm 35.27$ \\
& Makoka & $79.60 \pm 13.61$ \\
cowpea/maize intercropping & Chitedze & $181.02 \pm 35.21$ \\
& Makoka & $64.34 \pm 9.95$ \\
Nitrogen Levels & & \\
N0 & Chitedze & $75.35 \pm 16.39$ \\
& Makoka & $36.75 \pm 8.59$ \\
N1 & Chitedze & $136.63 \pm 27.61$ \\
& Makoka & $70.09 \pm 13.72$ \\
N2 & Chitedze & $180.17 \pm 34.55$ \\
& Makoka & $98.65 \pm 13.72$ \\
N3 & Chitedze & $259.98 \pm 39.85$ \\
& Makoka & $136.31 \pm 15.81$ \\
\hline
\end{tabular}

Key: $\mathrm{N} 0=0 \mathrm{~kg} \mathrm{~N} \mathrm{ha}^{-1}, \mathrm{~N} 1=52.5 \mathrm{~kg} \mathrm{~N} \mathrm{ha}^{-1}, \mathrm{~N} 2=78.75 \mathrm{~kg} \mathrm{~N} \mathrm{ha}^{-1}, \mathrm{~N} 3=105 \mathrm{~kg} \mathrm{~N} \mathrm{ha}^{-1}$.

Table 9. Effect of the interaction between nitrogen levels $\mathrm{x}$ cropping system $\mathrm{x}$ location on nutrient uptake in maize (means \pm std. error)

\begin{tabular}{|c|c|c|c|}
\hline Cropping System & Nitrogen levels & Location & Nutrient uptake (mg N/plant) \\
\hline \multirow[t]{8}{*}{ maize monocropping } & N0 & Chitedze & $64.98 \pm 8.73$ \\
\hline & & Makoka & $41.15 \pm 18.33$ \\
\hline & N1 & Chitedze & $147.37 \pm 39.42$ \\
\hline & & Makoka & $99.62 \pm 31.73$ \\
\hline & $\mathrm{N} 2$ & Chitedze & $136.78 \pm 29.40$ \\
\hline & & Makoka & $128.00 \pm 22.43$ \\
\hline & N3 & Chitedze & $258.27 \pm 72.06$ \\
\hline & & Makoka & $180.86 \pm 29.32$ \\
\hline \multicolumn{4}{|c|}{ bean/maize intercropping } \\
\hline & No & Chitedze & $59.12 \pm 31.09$ \\
\hline & & Makoka & $40.20 \pm 16.51$ \\
\hline & N1 & Chitedze & $97.79 \pm 37.84$ \\
\hline & & Makoka & $62.10 \pm 10.02$ \\
\hline & $\mathrm{N} 2$ & Chitedze & $223.72 \pm 79.01$ \\
\hline & & Makoka & $85.40 \pm 33.23$ \\
\hline & N3 & Chitedze & $244.27 \pm 78.38$ \\
\hline & & Makoka & $130.71 \pm 18.60$ \\
\hline \multicolumn{4}{|c|}{ cowpea/maize intercropping } \\
\hline & No & Chitedze & $101.94 \pm 40.52$ \\
\hline & & Makoka & $28.91 \pm 15.18$ \\
\hline & N1 & Chitedze & $164.75 \pm 70.40$ \\
\hline & & Makoka & $48.55 \pm 21.28$ \\
\hline & $\mathrm{N} 2$ & Chitedze & $179.99 \pm 72.97$ \\
\hline & & Makoka & $82.56 \pm 1.71$ \\
\hline & N3 & Chitedze & $277.41 \pm 86.27$ \\
\hline & & Makoka & $97.35 \pm 5.18$ \\
\hline
\end{tabular}

Key: N0 $=0 \mathrm{~kg} \mathrm{~N} \mathrm{ha}^{-1}, \mathrm{~N} 1=52.5 \mathrm{~kg} \mathrm{~N} \mathrm{ha}^{-1}, \mathrm{~N} 2=78.75 \mathrm{~kg} \mathrm{~N} \mathrm{ha}^{-1}, \mathrm{~N} 3=105 \mathrm{~kg} \mathrm{~N} \mathrm{ha}^{-1}$

\subsection{Nutrient Use Efficiency (NUE) of Maize}

The results in table 10 indicate that $\mathrm{N} 2\left(78.75 \mathrm{~kg} \mathrm{~N} \mathrm{ha}^{-1)}\right.$ had the highest nitrogen use efficiency followed by N1 
$\left(52.5 \mathrm{~kg} \mathrm{~N} \mathrm{ha}^{-1}\right)$ and $\mathrm{N} 3\left(105 \mathrm{~kg} \mathrm{~N} \mathrm{ha}^{-1}\right)$ in that order.

Table 10. Nutrient Use Efficiency of maize at different nitrogen levels

\begin{tabular}{lll}
\hline Nitrogen level & Maize yield(Mt/ha) & NUE (\%) \\
\hline N0 & 1.54 & \\
N1 & 3.99 & 46.67 \\
N2 & 5.37 & 48.63 \\
N3 & 6.25 & 44.86 \\
\hline
\end{tabular}

\subsection{Effect of Cropping System, Nitrogen Level, Location and Their Interaction on Legume Biomass and Number of Nodules}

The main effect cropping systems on legume DM yield and number of nodules was significant $(\mathrm{P}<0.05)$. The results in table 11 indicate that significantly higher values of legume DM yield were obtained under sole cowpea while significantly higher values of number of nodules were recorded under sole bean and bean/maize intercropping. On the other hand significantly lower values of legume DM yield were recorded under bean/maize intercropping system while significantly lower values of number of nodules were recorded under sole cowpea. From the same table the main effect of location was significant $(\mathrm{P}<0.05)$ for $\mathrm{DM}$ yield, and number of nodules. Significantly higher number of nodules was reported at Chitedze. On the other hand significantly higher values of DM yield were recorded under Makoka.

The interactive effect of cropping systems $x$ levels of nitrogen was significant $(\mathrm{P}<0.05)$ on legume DM yield and number of nodules (Table 12). Significantly higher values were obtained under the following treatment combinations: cowpea/maize intercropping $\mathrm{x} \mathrm{N} 3$ and bean/maize intercropping $\mathrm{x} \mathrm{N} 1$, respectively. On the other hand significantly lower values were obtained under the following treatment combinations; bean/maize intercropping system x N2 and cowpea/maize intercropping x N2 for legume DM and number of legumes, respectively (Table 13).

The effect of interaction of cropping system $x$ nitrogen level $x$ location was significant $(P<0.05)$ on legume dry matter (Table 14). From the same table it was observed that the interaction was significant under cowpea/maize intercropping and it was not significant under bean/maize intercropping system. The interactions that produced significantly higher values of legume DM and number of nodules were observed under the following treatment combinations; cowpea/ maize intercropping x N3 x Makoka, and cowpea/maize intercropping x N3 x Chitedze.

The effect of the interaction of cropping system $x$ location was significant for legume DM yield (Table 15). The results indicated that significantly higher values of legume yield DM yield were obtained under the treatment combination of sole cowpea x Makoka. From the same table significantly higher values for number of nodules were obtained under the following treatment combinations; cowpea/maize intercropping $\mathrm{x}$ Chitedze. The interaction of nitrogen levels $\mathrm{x}$ location was significant for legume yield DM yield (Table 15). Significantly higher values were obtained under the following treatment combinations; N3 level of nitrogen $x$ Makoka.

Table 11. Effect of Nitrogen level, cropping system and location on legume dry matter (DM) yield and number of nodules

\begin{tabular}{lll}
\hline Treatments & Legume DM yield (Mt/ha) & Number of nodules/plant \\
\hline Nitrogen Level & & \\
N0 & $1.00 \mathrm{a}$ & $14.58 \mathrm{a}$ \\
N1 & $1.05 \mathrm{a}$ & $12.08 \mathrm{a}$ \\
N3 & $1.16 \mathrm{a}$ & $13.75 \mathrm{a}$ \\
N2 & $0.93 \mathrm{a}$ & $9.08 \mathrm{a}$ \\
Cropping system & & \\
Sole cowpea & $2.66 \mathrm{a}$ & $5.66 \mathrm{c}$ \\
Sole bean & $1.75 \mathrm{~b}$ & $26.33 \mathrm{a}$ \\
cowpea/maize intercropping & $1.26 \mathrm{c}$ & $7.33 \mathrm{bc}$ \\
bean/maize intercropping & $0.81 \mathrm{~d}$ & $17.41 \mathrm{ab}$ \\
Location & & \\
Chitedze & $0.98 \mathrm{~b}$ & $19.40 \mathrm{a}$ \\
Makoka & $1.56 \mathrm{a}$ & $6.80 \mathrm{~b}$ \\
\hline
\end{tabular}

Means with the same letter within a column are not significantly different $(\mathrm{P}<0.05)$ according to Duncan test

Key: $\mathrm{N} 0=0 \mathrm{~kg} \mathrm{~N} h a^{-1}, \mathrm{~N} 1=52.5 \mathrm{~kg} \mathrm{~N} \mathrm{ha}^{-1}, \mathrm{~N} 2=78.75 \mathrm{~kg} \mathrm{~N} \mathrm{ha}^{-1}, \mathrm{~N} 3=105 \mathrm{~kg} \mathrm{~N} \mathrm{ha}^{-1}$. 
Table 12. Effect of the interaction of cropping systems $x$ levels of nitrogen on legume DM yield, and number of nodules (means \pm std. error)

\begin{tabular}{llll}
\hline Cropping System & Nitrogen Level & DM Yield(Mt/ha) & Number of Nodules/plant \\
\hline bean/maize intercropping & & & \\
& N0 & $0.83 \pm 0.08$ & $18.83 \pm 6.54$ \\
& N1 & $0.87 \pm 0.17$ & $19.00 \pm 6.10$ \\
& N2 & $0.65 \pm 0.09$ & $13.67 \pm 2.74$ \\
& N3 & $0.88 \pm 0.16$ & $18.17 \pm 10.84$ \\
cowpea/maize intercropping & & & $10.33 \pm 1.36$ \\
& N0 & $1.17 \pm 0.24$ & \\
& N1 & $1.4 \pm 0.22$ & $5.17 \pm 0.65$ \\
& N2 & $1.21 \pm 0.31$ & $4.50 \pm 0.89$ \\
& N3 & $1.45 \pm 0.29$ & $9.33 \pm 5.94$ \\
\hline $\mathrm{kg} \mathrm{N} \mathrm{ha}^{-1}, \mathrm{~N} 1=52.5 \mathrm{~kg} \mathrm{~N} \mathrm{ha}^{-1}, \mathrm{~N} 2=78.75 \mathrm{~kg} \mathrm{~N} \mathrm{ha}^{-1,} \mathrm{~N} 3=105 \mathrm{~kg} \mathrm{~N} \mathrm{ha}^{-1}$
\end{tabular}

Table 13. Effect of interaction of cropping system x nitrogen level x location on legume DM yield, and number of nodules (means \pm std. error)

\begin{tabular}{|c|c|c|c|c|}
\hline Cropping System & Nitrogen Level & Location & DM (Mt/ha) & Number of nodules/plant \\
\hline \multicolumn{5}{|c|}{ bean/maize intercropping } \\
\hline & No & Chitedze & $0.94 \pm 0.04$ & $31.00 \pm 7.94$ \\
\hline & & Makoka & $0.72 \pm 0.13$ & $6.67 \pm 1.67$ \\
\hline & N1 & Chitedze & $0.86 \pm 0.15$ & $32.00 \pm 4.04$ \\
\hline & & Makoka & $0.88 \pm 0.34$ & $6.00 \pm 1.00$ \\
\hline & $\mathrm{N} 2$ & Chitedze & $0.76 \pm 0.09$ & $19.67 \pm 0.88$ \\
\hline & & Makoka & $0.55 \pm 0.15$ & $7.67 \pm 0.88$ \\
\hline & N3 & Chitedze & $0.83 \pm 0.19$ & $31.33 \pm 20.34$ \\
\hline & & Makoka & $0.94 \pm 0.31$ & $5.00 \pm 0.58$ \\
\hline \multicolumn{5}{|c|}{ cowpea/maize intercropping } \\
\hline & No & Chitedze & $0.89 \pm 0.23$ & $10.33 \pm 1.86$ \\
\hline & & Makoka & $1.44 \pm 0.41$ & $10.33 \pm 2.40$ \\
\hline & N1 & Chitedze & $0.85 \pm 0.19$ & $5.00 \pm 1.15$ \\
\hline & & Makoka & $1.62 \pm 0.26$ & $5.33 \pm 0.88$ \\
\hline & $\mathrm{N} 2$ & Chitedze & $0.66 \pm 0.06$ & $3.33 \pm 0.67$ \\
\hline & & Makoka & $1.75 \pm 0.44$ & $5.67 \pm 1.45$ \\
\hline & N3 & Chitedze & $1.10 \pm 0.15$ & $14.67 \pm 12.17$ \\
\hline & & Makoka & $1.79 \pm 0.53$ & $4.00 \pm 0.00$ \\
\hline
\end{tabular}

Key: $\mathrm{N} 0=0 \mathrm{~kg} \mathrm{~N}^{-1}, \mathrm{~N} 1=52.5 \mathrm{~kg} \mathrm{~N} \mathrm{ha}^{-1}, \mathrm{~N} 2=78.75 \mathrm{~kg} \mathrm{~N} \mathrm{ha}^{-1}, \mathrm{~N} 3=105 \mathrm{~kg} \mathrm{~N} \mathrm{ha}^{-1}$ 
Table 14. Effect of the interactions between cropping systems and location, nitrogen levels and location on legume DM yield, and number of nodules (means \pm std. error)

\begin{tabular}{llll}
\hline Treatments & Location & DM (Mt/ha) & Number of Nodules/plant \\
\hline $\begin{array}{l}\text { Cropping System } \\
\text { bean/maize intercropping }\end{array}$ & & & \\
& & & \\
& Chitedze & $0.84 \pm 0.06$ & $28.50 \pm 4.98$ \\
cowpea/maize intercropping & Makoka & $0.77 \pm 0.12$ & $6.33 \pm 0.56$ \\
& & & \\
& Chitedze & $0.88 \pm 0.09$ & $8.33 \pm 2.97$ \\
cowpea monocropping & Makoka & $1.65 \pm 0.18$ & $6.33 \pm 0.96$ \\
& & & \\
& Chitedze & $1.84 \pm 0.39$ & $5.67 \pm 3.18$ \\
bean monocropping & Makoka & $3.47 \pm 1.00$ & $5.67 \pm 1.76$ \\
& & & \\
& Chitedze & $1.11 \pm 0.08$ & $41.00 \pm 13.89$ \\
Nitrogen level & Makoka & $2.39 \pm 0.83$ & $11.67 \pm 2.40$ \\
$\mathrm{~N} 0$ & & & \\
& Chitedze & $0.92 \pm 0.11$ & $20.67 \pm 5.89$ \\
$\mathrm{~N} 1$ & Makoka & $1.08 \pm 0.25$ & $8.50 \pm 1.54$ \\
& Chitedze & $0.86 \pm 0.15$ & $18.50 \pm 6.32$ \\
$\mathrm{~N} 2$ & Makoka & $1.25 \pm 0.25$ & $5.67 \pm 0.61$ \\
& Chitedze & $0.71 \pm 0.05$ & $11.50 \pm 3.69$ \\
$\mathrm{~N} 3$ & Makoka & $1.15 \pm 0.34$ & $6.67 \pm 0.88$ \\
& Chitedze & $0.97 \pm 0.18$ & $23.00 \pm 11.24$ \\
$\mathrm{~kg} \mathrm{~N}^{-1}, \mathrm{~N} 1=52.5 \mathrm{~kg} \mathrm{~N}^{-1}{ }^{-1}$ N2 $=78.75 \mathrm{~kg} \mathrm{~N} \mathrm{ha}{ }^{-1}, \mathrm{~N} 3=105 \mathrm{~kg} \mathrm{~N} \mathrm{ha}^{-1}$ &
\end{tabular}

\section{Discussion}

\subsection{Main Effects of Cropping System, Nitrogen Level, Location on Maize Grain Yield}

$\mathrm{N}$ fertilizer application $\left(52.5,78.75\right.$ and $\left.105 \mathrm{~kg} \mathrm{~N}^{-1}\right)$ resulted in significant increases in maize grain yield compared to control (no fertilizer). Nitrogen is a critical macronutrient for the growth of maize and its application enhances vigorous vegetative growth (Havlin et al., 2008). Many authors including Sebetha (2015), Abayomi, George-Arijenja and Kolawole (2006), Mahdi and David (2005), Morgado and Willey (2003) and Muchow (1988), similarly reported that application of $\mathrm{N}$ fertilizer generally resulted in increases in maize grain yield. Significant increases in maize grain yields occurred with increase in $\mathrm{N}$ level, from 0 to $105 \mathrm{~kg} \mathrm{~N} \mathrm{ha}^{-1}$, an indication that application of $105 \mathrm{~kg} \mathrm{~N} \mathrm{ha}^{-1}$ was optimal for maximum yield per unit area. Elevated $\mathrm{N}$ concentration results to healthier plant growth (Legg, Stanford \& Bennett, 1979: Meisinger, Bandel, Stanford, \& Legg, 1985). The reduction in maize grain yield under maize without $\mathrm{N}$ fertilizer is in agreement with the findings of Ding et al., (2005) and Lucas (1986).

Maize grain yields were not significantly increased by inclusion of bean and cowpea. Maize has a C4 carbon assimilation pathway and may have had a competitive edge over legumes, which are C3 plants (Kitonyo, Chemining'wa, \& Muthomi, 2013; Sage \& Zhu, 2011). In addition, maize was more competitive for soil nitrogen because its roots are distributed in both shallow and deeper layers (Carruthers et al., 2000). This is in contrast to the root systems of legumes which are smaller and confined to the upper layers (Hauggaard-Nielsen, Ambus \& Jensen, 2001). Contrarily, most studies report that intercropped systems yield more than maize monocropping due to the ability of intercropped legumes to fix most of their nitrogen from the atmosphere (Chabi-Olaye, Nolte, Schulthess, \& Borgemeister, 2005; Hauggaard-Nielson \& Jensen, 2001). Common beans are, however, poor $\mathrm{N}$ fixers, in comparison to other legumes; hence they do not contribute significantly towards the N requirement by maize (Westermann, Kleinkopf, Porter, \& Leggett, 1981, Bliss, 1993; Martinez-Romero, 2003). Mineral nitrogen may have reduced the rate of nitrogen fixation by cowpea in this study (Houwaard, 1979).

Results showed significantly higher maize grain yield at Chitedze than Makoka. This may be attributed to lower rainfall amounts and higher temperature at Makoka. The optimal temperature for warm season maize is $15-20{ }^{\circ} \mathrm{C}$ for planting and $20-30{ }^{\circ} \mathrm{C}$ for the regular growing season (Bird, Cornelius, \& Keys, 1977). The 
temperature range for Makoka during the growing season was 17.84- $28.06{ }^{\circ} \mathrm{C}$ (Table 1). The higher temperature led to higher rates of evapotranspiration and therefore increased competition for moisture (Ben-Asher, Garcia, Garcia, \& Hoogenboom, 2008). Water forms an integral part of plant body and plays an important role in growth initiation, maintenance of developmental process of plant life and hence has pivotal function in crop production (Aslam et al., 2012).

\subsubsection{Interaction Effects of Cropping System, Nitrogen Level, Location on Maize Grain Yield}

The interaction effect of cropping systems $\times \mathrm{N}$ level was not significant for maize grain yield. This may possibly have been due to inhibition of symbiotic nitrogen fixation by the legumes due the application of $\mathrm{N}$ fertilizer (Erker \&Brick, 2014). This is in agreement with the findings of Omokanye, Kelleher, and McInnes, (2013) who reported that the interaction effect of cropping system $\mathrm{x}$ nitrogen levels on maize grain yield was not significant. The results further indicated that maize grain yield was not significantly affected by the interaction of cropping system $\mathrm{x}$ location because maize faced stiff competition for resources from legumes. These findings are contrary to the findings of Sebetha (2015) who reported that the interaction had a significant effect on maize DM yield.

The interaction effect of cropping system $\mathrm{x}$ location $\mathrm{x}$ nitrogen level on maize grain yield was not significant. These findings suggest that cropping system did not play a vital role in dry matter accumulation. This finding is not in agreement with Sebetha (2015), who reported the significance of the interaction on maize grain yield.

\subsection{Main Effects of Cropping System, Nitrogen Level, and Location On Nitrogen Uptake By Maize}

The results indicated that an increase in $\mathrm{N}$ uptake by maize occurred with increasing rates of $\mathrm{N}$ fertilizer applied. The maximum value (198.15 mg-N/plant) was recorded with the highest $\mathrm{N}$ fertilizer level $\left(105 \mathrm{~kg} \mathrm{~N} \mathrm{ha}^{-1}\right)$ while the lowest (56.05 mg-N/plant) at zero fertilizer rate. Rahman (2011) and Morgado and Willey (2003) similarly reported that $\mathrm{N}$ uptake by maize plant was influenced significantly by $\mathrm{N}$ fertilizer application rate. They reported lowest uptake in control (no N fertilizer) treatment. Chirnogeanu, Badea, Petcu, and Picu (1997) also documented that high levels of soil nitrogen had a significant positive influence on the nutrient uptake and translocation in leaves. Thus, the $\mathrm{N}$ content in maize plants increased in variation with high fertilizer rates.

The results of this study indicated that there were no significant differences in nutrient uptake by maize in the different cropping systems. Common bean being a poor $\mathrm{N}$ fixer did not supply enough $\mathrm{N}$ to be taken up by both the legume and the cereal crop involved. Application of nitrogen fertilizer might have hindered symbiotic nitrogen fixation by cowpea (Henson \& Bliss, 1991; Erker \& Brick, 2014). Contrarily, Eskandari and Ghanbari (2009) reported significantly greater nitrogen uptake in intercropping than sole maize. They reported that intercropping was more efficient at exploiting a larger soil total volume if component crops have different rooting habits, especially depth of rooting.

Nitrogen uptake by maize was significantly greater at Chitedze than at Makoka. This difference may be attributed to the differences in total soil $\mathrm{N}$ at the sites. The levels at Chitedze and Makoka were medium $(0.20 \%)$ and low $(0.10 \%)$, respectively (Table 2), according to the nutrient classification of Landon (1991).

\subsubsection{Interaction Effects of Cropping System, Nitrogen Level, Location on Nitrogen Uptake by Maize}

The effect of cropping system $\times \mathrm{N}$ level interaction on $\mathrm{N}$ uptake by maize was significant. Significantly higher $\mathrm{N}$ uptake by maize was observed in the bean/maize intercropping system than cowpea/maize intercropping under $\mathrm{N} 3$ level of N, in both cropping systems, and maize monocropping combined with nitrogen levels of N1 and N2. This might have been due to enhanced supply nitrogen from fertilizer. Additionally, there may have occurred greater exploitation of a larger soil total volume for nutrients and water due to different rooting habits of the component crops in an intercropping system.

The effect of cropping systems $\times$ location interaction on $\mathrm{N}$ uptake in maize was significant only under maize monocropping system. The higher uptake in maize monocropping system at both sites was because the maize did not face competition for nutrients from legumes, as they were sole crops.

$\mathrm{N}$ uptake by maize was not significantly affected by the interaction of nitrogen levels $\times$ location. It is possible that this finding might be influenced by the location conditions such as moisture, soil aeration, soil drainage and soil textures which have an impact on $\mathrm{N}$-transport and $\mathrm{N}$-transformation processes that limit $\mathrm{N}$ availability to crops or lead to losses such as through leaching.

The nitrogen level $\times$ cropping system $\times$ location interaction effect on $\mathrm{N}$ uptake by maize was significant. The higher uptake observed under combination of maize monocropping system, N3 level of nitrogen at Chitedze was because sole maize did not have any competition from legumes for resources such as nutrients. $\mathrm{N}$ uptake was significantly lower under cowpea/maize intercropping system combined with N0 level of nitrogen at Makoka 
research station. It is possible that maize suffered stiff competition for resources from legumes.

\subsection{Nutrient Use Efficiency (NUE) of Maize}

Maximum maize NUE $(48.63 \mathrm{~kg} / \mathrm{kg})$ was obtained at application rate of $78.75 \mathrm{~kg} \mathrm{~N}^{-1}$ and the minimum value $(44.86 \mathrm{~kg} / \mathrm{kg})$ was recorded at the highest $\mathrm{N}$ rate $\left(105 \mathrm{~kg} \mathrm{~N} \mathrm{ha}^{-1}\right)$. The decrease in NUE with increasing $\mathrm{N}$ fertilizer rate was because yield rises less than the $\mathrm{N}$ supply in soil and fertilizer (Lopez-Bellido \& Lopez-Bellido (2001), Raun \& Johnson (1999), Pierce \& Rice (1988), Sowers, Pan, Miller, \& Smith (1994) and Zhao et al., (2006) also reported that high rates of N decreased NUE in cereals. The findings of Kanampiu, Raun and Johnson (1997) generally indicated decreases in NUE but increases grain protein content and $\mathrm{N}$ loss with increasing $\mathrm{N}$ fertilizer rate.

\subsection{Main Effects of Cropping System, Nitrogen Level, Location on Legume DM Yield and Number of Nodules}

The main effect of cropping system on legume DM yield, and number of nodules was significant. Higher values of legume DM yield were reported under sole cowpea $(2.66 \mathrm{Mt} / \mathrm{ha})$ and sole bean $(1.75 \mathrm{Mt} / \mathrm{ha})$ while lower values were obtained under cowpea/maize intercropping $(1.26 \mathrm{Mt} / \mathrm{ha})$ and bean/maize intercropping system $(0.81 \mathrm{Mt} / \mathrm{ha})$.These findings might be attributed to the well-known idea that cereals take up nutrients, especially $\mathrm{N}$, mainly during the vegetative growth stage and associated vigorous growth may cause shading of the legume and thereby reduce its growth during later growth stages resulting in low yielding ability (Banik et al., 2006). These results are supported by the findings of Birteeb, Addah, Jakper, and Addo-Kwafo (2011) who reported significantly reduced legume DM yield of intercropped legumes.

The main effect of $\mathrm{N}$ level on legume DM yield and number of nodules was not significant. These findings may be attributed to the fact that application of mineral nitrogen reduces both nodulation and the rate of nitrogen fixation by legumes (Houwaard, 1979). These findings are in agreement with the findings of Bagayoko, Buekert, Lung, Bationo and Romheld (1996) who observed that cowpea DM yield was not influenced by $\mathrm{N}$ application. Legume number of nodules per plant were higher under $\mathrm{N} 0\left(0 \mathrm{~kg} \mathrm{~N} \mathrm{ha}^{-1}\right)$ than $52.5,78.75$ and $105 \mathrm{~kg} \mathrm{~N} \mathrm{ha}^{-1}$, however, they were not significantly different from the parameters obtained under $0 \mathrm{~kg} \mathrm{~N} \mathrm{ha}^{-1}$. It was expected that legume DM yield, and number of nodules under $\mathrm{N}$ fertilizer application would be lower than the control since $\mathrm{N}$ fertilization has a negative effect of legume growth, development and yield.

Legume DM yield was significantly affected by location. Legume DM yield was higher at Makoka than at Chitedze. It was also observed that the number of nodules per legume plant was affected by the location of the study site. Legumes planted at Chitedze research station had significantly higher number of nodules per plant (19.40) than at Makoka (6.80). This may be attributed to different soil structure and climatic conditions of these two sites. On the other hand $\mathrm{N}$ fertilizer application has a well-established negative effect on nitrogen fixation of legume root nodules. It is also reported that with increasing doses of $\mathrm{N}$ there is a nearly linear decrease in the number of root nodule (Becker, Alazard, \& Ottow, 1986) This finding concurs with what Sebetha (2015) observed. He reported that number of nodules per cowpea plant was affected by location. The effect of location on number of number nodules can be attributed to fluctuations in $\mathrm{pH}$, nutrient availability, temperature, and water status, among other factors that greatly influence the growth, survival, and metabolic activity of nitrogen fixation bacteria and plants, and their ability to enter into symbiotic interactions (Werner \& Newton, 2005).The results on the number of nodules per plant concur with the ranges reported by Bhuvaneswari, Lesniak, and Bauer (1998).

4.4.1 Interaction Effects of Cropping System, Nitrogen Level, Location on Legume DM Yield and Number of Nodules

Legume DM yields and number of nodules were significantly affected by the interaction effect of cropping systems $\times$ levels of nitrogen. Findings of the study agree with the study of Sebetha (2015) who reported that the interaction of nitrogen and location significantly affected the number of nodules per cowpea plant. In addition common beans are poor $\mathrm{N}$ fixers, in comparison to other legumes; hence they do not contribute significantly towards the N requirement by maize (Westermann et al., 1981, Bliss, 1993; Martinez-Romero, 2003).

Significantly higher values were obtained under the following treatment combinations:, cowpea/maize intercropping x N3, and bean/maize intercropping x N1, respectively. On the other hand significantly lower values were obtained under the following treatment combinations; bean/maize intercropping system x N2 and cowpea/maize intercropping x N2 for legume DM yield and number of nodules, respectively. This might be attributed to cowpea having a higher potential of fixing $\mathrm{N}$ from the atmosphere through biological nitrogen fixation than beans (Freitas, Sampaio, Santos, \& Fernandes, 2010).

The interaction effect of cropping system $\times$ location was significant for legume DM yield. The results indicate 
that significantly higher values of legume DM yield were obtained under the treatment combination of sole cowpea x Makoka. This might be attributed to good environmental factors present at Makoka such conducive as temperature, good moisture content and good soil structure which were conducive for the production of cowpeas. Legume DM yield was significantly affected by the interaction effect of nitrogen levels and location. These findings contributed to the significance of comparing cropping systems towards improvement of legume yields since such interaction effect on legume yield was not revealed during previous studies.

The interaction effect of cropping system $\mathrm{x}$ nitrogen level $\mathrm{x}$ location was significant on legume DM yield. The interaction was however significant for number of nodules per cowpea plant (cowpea/maize intercropping).This is in agreement with what was reported by Sebetha (2015) that the interaction significantly affected the number of nodules per cowpea plant.

\section{Conclusions}

Diminishing land sizes, due to the ever increasing human population, and continuous cultivation practices have led to declining soil fertility and maize yield in Malawi. The study to determine effects of mineral $\mathrm{N}$ fertilizer application and legume integration on maize nutrient uptake and yield, demonstrated that $\mathrm{N}$ absorption by maize occurred with application of the water soluble urea fertilizer. $\mathrm{N}$ uptake increased with increase in fertilizer rate. Higher values were obtained with the application of $105 \mathrm{~kg} \mathrm{~N}^{-1}$. The integration of legumes did not have any significant effect on uptake. This may have been because $\mathrm{N}$ fixation was not optimal. Nodulation in cowpea and common bean, a major conduit for available nitrogen into the biosphere, was not significantly increased by $\mathrm{N}$ application. Additionally, common bean is a poor $\mathrm{N}$ fixer. The study further showed that the effect of cropping system $\times \mathrm{N}$ level interaction on N uptake by maize was significant. Significantly higher values were recorded under cowpea/maize intercropping system and N3 nitrogen level $\left(105 \mathrm{~kg} \mathrm{~N} \mathrm{ha}^{-1}\right)$. It was observed that NUE decreased with increasing $\mathrm{N}$ fertilizer rate. The minimum value $(44.86 \mathrm{~kg} / \mathrm{kg})$ was recorded at the highest $\mathrm{N}$ rate $\left(105 \mathrm{~kg} \mathrm{~N} \mathrm{ha}^{-1}\right)$. Most of the fertilizer remains unutilized leading to nutrient toxicity. On the other hand, there was a tremendous reduction in maize development and yield when no $\mathrm{N}$ fertilizer was applied to the maize. Cropping system did not have an effect on maize development and yield..

\section{Acknowledgments}

This material is based upon work supported by the United States Agency for International Development, as part of the Feed the Future initiative, under the CGIAR Fund, award number BFS-G-11-00002, and the predecessor fund the Food Security and Crisis Mitigation II grant, award number EEM-G-00-04-00013. We also thank the Officers in charge of the Chitedze and Makoka research stations for providing research fields.

\section{References}

Abayomi, Y., George-Arijenja, A., \& Kolawole, I.A. (2006). Comparative leaf growth and grain yield responses of hybrid and open-pollinated maize genotypes to nitrogen fertilizer application. Agro Search, 8(1 and 2): 13-26. http://dx.doi.org/10.4314/agrosh.v8i1.39435

Anderson, J. M., \& Ingram, J. S. I. (1993). Tropical Soil Biology and Fertility. A hand book of Methods. 2ended. Wallingford, UK; CABI Publishing.

Aslam, M., Zamir, M. S. I., Afzal, I., Yasee, M., Mubeen, M., \& Shoaib, A. (2012). Drought stress, its effect on maize production and development of drought tolerance through potassium application. Agronomical Research in Moldavia, 2(154).

Bagayako, M., Buekert, A., Lung, G., Bationo, A., \& Romheld, V. (2000). Cereal/legume rotation effects on cereal growth in Sudano-Sahelian West Africa: Soil mineral nitrogen, mycorrhizae and nematodes. Plant Soil, 218, 103-116. https://doi.org/10.1023/A:1014957605852

Banik, P., Midya, A., Sarkar, B. K., \& Ghose, S. S. (2006). Wheat and chickpea intercropping systems in an additive series experiment: Advantages and weed smothering. European Journal of Agronomy, 24, 325-332. https://doi.org/10.1016/j.eja.2005.10.010

Becker, M., Alazard, D., \& Ottow, J. C. G. (1986). Mineral nitrogen effect on nodulation and nitrogen fixation of the stem-nodulating legume Aeschynomene afraspera. Z. Pflanzenernaehr. Bodenk, 149, 485-491. https://doi.org/10.1002/jpln.19861490411

Ben-Asher, J., Garcia A., Garcia, Y., \& Hoogenboom, G. (2008). Effect of high temperature on photosynthesis and transpiration of sweet corn (Zea mays L. var. rugosa). Photosynthetica, 46(4), 595-603. https://doi.org/10.1007/s11099-008-0100-2

Bhuvaneswari, T. Y, Lesniak, A. P., \& Bauer, W. D. (1998). Efficiency of nodule initiation in cowpea and 
soybean. Plant Physiology Journal, 86, 1210-1215. https://doi.org/10.1104/pp.86.4.1210

Bird, I. F., Cornelius, M. J., \& Keys, A. J. (1977). Effects of temperature on photosynthesis by maize and wheat. Journal of Experimental Botany, 28, 519524. https://doi.org/10.1093/jxb/28.3.519

Birteeb, P. T., Addah, W., Jakper, N., \& Addo-Kwafo, A. (2011). Effects of intercropping cereal-legume on biomass and grain yield in the savannah zone. Livestock Research for Rural Development Journal, 23(198).

Bliss, F. A. (1993). Breeding common bean for improved biological nitrogen fixation. Plant Soil Journal, 152, 71-79.

Brentrup, F., \& Palliere, C. (2010). Nitrogen use efficiency as an agro-environmental indicator. OECD workshop

Carruthers, K., Prithiviraj, B., Fe, O., Cloutler, D., Martin, R. C., \& Smith, D. L. (2000). Intercropping corn with soybean, lupin and forages: yield component responses. European Journal of Agronomy, 12, 163-115. https://doi.org/10.1016/s1161-0301(99)00051-9

Chabi-Olaye, A., Nolte, C. F., \& Borgemeister, C. (2005). Relationships of intercropped maize, stem borer damage to maize yield and land-use efficiency in the humid forest of Cameroon. Bulletin of Entomological Research, 95, 417-427. https://doi.org/10.1079/ber2005373

Chirnogeanu I., Badea, E Petcu E., \& Picu I. (1997). The effect of mineral fertilization on uptake of some nutrients in maize under irrigated conditions. Romania: Romanian Agricultural Research, 55-66

Ding, L., Wang, K. J., Jiang, G. M., Biswas, D. K., Xu, H., Li, L. F., \& Li, Y. H. (2005). Effect of nitrogen deficiency on photosynthetic traits of maize hybrids released in different years. Annals of Botany, 96(5), 925-930. https://doi.org/10.1093/aob/mci244

Erker, B., \& Brick, M. A. (2014). CSU Homepage. Retrieved November 05, 2014, from Colorado State University Extension: http:www.ext.colostate.edu/pubs/crops/00305.html

Eskandari, H., \& Ghanbari, A. (2009). Intercropping of maize (Zea mays) and cowpea (Vigna sinensis) as whole-crop forage: Effect of different planting pattern on total dry matter production and maize forage quality. Notulae Botanicae Horti Agrobotanici Cluj-Napoca, 37(2), 152-155.

FAOSTAT. (2013). Cowpea. Retrieved from http://faostat.fao.org/cowpea.

Food \& Agriculture Organization (FAO) of the United Nations. (1999). Phaseolus Bean: Post-harvest operations

Food \& Agriculture Organization (FAO) of the United Nations. (2004). COWPEA: Post-harvest operations

Freitas, A.D.S. Sampaio, E.V.S.B. Santos, C.E.R.S.\& Fernandes, A.R.(2010). Biological nitrogen fixation in legume trees of the Brazilian caatinga. Journal of Arid Environments, 74:344 - 349. https://doi.org/10.1016/j.jaridenv.2009.09.018

Gachene, C. K. K., \& Kimaru, G. (2003). Soil fertility and land productivity: A guide for extension workers in the eastern region of Africa RELMA Technical Handbook Series 30. Nairobi, Kenya

Hauggaard-Nielsen, H., \& Jensen E. S. (2001). Evaluating pea and barley cultivars for complementarity in intercropping at different levels of soil N availability. Field Crops Research, 72, 185-196. http://dx.doi.org/10.1016/S0378-4290 (01)00176-9

Hauggaard-Nieson, H., Ambus, P., \& Jensen, E. S. (2001). Temporal and spatial distribution of roots and competition for nitrogen in pea-barley intercrops. A field studies employing $23 \mathrm{P}$ techniques. Plant and Soil, 236, 63-74. https://doi.org/10.1023/a:1011909414400

Havlin, J. L., Tisdale, S. L, Beaton, J. D., \& Werner, L. N. (2008). Soil Fertility and Fertilizers. An introduction to Nutrient Management. Seventh edition. Pearson Education, Inc, Publishing. Prentice Hall

Hayat, R., Safdar, A., Siddique, M. T., \& Chatha, T. H. (2008). Biological Nitrogen Fixation of Summer Legumes and their Residual Effects on Subsequent Rain fed Wheat Yield. Pakistan Journal of Botany, 40(2), 711-722.

Henson, R. A., \& Bliss, F. A. (1991). Effects of $\mathrm{N}$ fertilizer application timing on common bean production. Fertilizer Research, 29, 133-138. https://doi.org/10.1007/bf01048951

Houwaard, F. (1979): Effect of combined nitrogen on symbiotic nitrogen fixation in pea plants. (PhD. Thesis, Agricultural University Wageningen, Netherlands.)

International Crops Research Institute for Semi-arid Tropics [ICRISAT] (2013). Bulletin of tropical legumes.

International Institute of Tropical Agriculture (IITA). (2016). http://www.iita.org/cowpea; jsessionid. Accessed 
on $20 / 03 / 2016$.

Kanampiu, F. K., Raun, W. R., \& Johnson, G. V. (1997). Effect of Nitrogen Rate on Plant Nitrogen Loss in Winter Wheat Varieties. Journal of Plant Nutrition, 20, 389-404. https://doi.org/10.1080/01904169709365259

Kitonyo, O. M., Chemining'wa, G. N., \& Muthomi, J. W. (2013). Productivity of farmer-preferred maize varieties intercropped with beans in semi-arid Kenya. International Journal of Agronomy and Agricultural Research, 3(1), 6-16.

Landon, J. R. (Ed.) (1991) .Booker Tropical Soil Manual. A Handbook for Soil Survey and Agricultural Land Evaluation in the Tropics and Subtropics. John Wiley and Sons, Inc., New York. 474pp.

Legg, J. O., Stanford, G., \& Bennett, O. L. (1979). Utilization of labelled N fertilizer by silage corn under conventional and no-till culture. Agronomy Journal, 71, 1009-1015. https://doi.org/10.2134/agronj1979.00021962007100060028x

Lopez-Bellido, R. J., \& Lopez-Bellido, L. (2001). Efficiency of nitrogen in wheat under Mediterranean conditions: effect of tillage, crop rotation and $\mathrm{N}$ fertilization. Field Crops Research, 71, 31-46. https://doi.org/10.1016/s0378-4290(01)00146-0

Lucas, E. O. (1986). The effect of density and nitrogen fertilizer on the growth and yield of maize (Zea mays L.) in Nigeria. The Journal of Agricultural Science, 107(3), 573- 578.

Magreta, R., \& Jambo, J. (2012). A critique on research priotisation on new bean markets and the youth in Malawi: Transforming the region. Paper prepared for the conference Young People, Farming and Food, 19-21 March 2012, Accra Ghana.

Mahdi, A. K., \& David, K. M. (2005). Effect of tillage system and nitrogen source on corn yield. Australia Society of Agronomy, 2(1), 04-13.

MAIFS (Ministry of Agriculture, Irrigation and Food Security) (2004). Guide to agricultural production and natural resources management in Malawi. Published and printed by Agricultural Communication Branch, Ministry of Agriculture, irrigation and Food Security Lilongwe, Malawi.

Martinez-Romero, E. (2003). Diversity of Rhizobium-Phaseolus vulgaris symbiosis: overview and perspectives. Plant and Soil, 252, 11-23.

Mbewe, E. C. (2011). The efficacy of Jatropha seed cake as organic manure (Unpublished master's thesis). University of Malawi, Bunda College.

Meisinger, J. J., Bandel, V. A., Stanford, G., \& Legg. J. O. (1985). Nitrogen utilization of corn under minimum tillage and moldboard plow tillage. I. Four years results using labelled $\mathrm{N}$ fertilizer on an Atlantic Coastal plain soil. Agronomy Journal, 77, 602-61. https://doi.org/10.2134/agronj1985.00021962007700040022x

Morgado, L. B., \& Willey, R. W. (2003). Effects of plant population and nitrogen fertilizer on yield and efficiency of maize-bean intercropping. Pesquisa Agropecuária Brasileira, 38(11). https://doi.org/10.1590/s0100-204x2003001100002

Muchow, R. C. (1988). Effect of nitrogen supply on the comparative productivity of maize and sorghum in a semi-arid tropical environment. Field Crops Research, 18, 1-16.

Munthali, M. W., \& Mazuma, E. D. (2010). Evaluation of TwinN as a source of nitrogen for maize production. Chitedze Agricultural Research Station, Malawi.

Nkongolo. K. K., Bokosi, J., Malusi, M., Vokhiwa, Z., \& Mphepo, M. (2009). Agronomic, culinary and genetic characterization of selected cowpea elite lines using farmer's breeder's knowledge: A case study from Malawi. African Journal of Plant Science, 3(7), 147-156.

Omokanye, A. T., Kelleher, F. M., \& McInnes, A. (2013). Crop residues for mulch, feed yield and quality as influenced by low-input maize-based cropping systems and N-fertilizer. Agricultural Journal, 8(5), 222-231.

Paul, E. A. (2008). Soil Microbiology, Ecology, and Biochemistry, $3^{\text {rd }}$ Edition. Academic Press, Boston.

Peterburgski, A. V. (1986). Practical Guidance on Agro-chemistry. Kolos Publ., Moscow.

Pierce, F. J., \& Rice, C. W. (1988). Crop rotation and its impact on efficiency of water and nitrogen use. In: Hargrove (ed) Cropping strategies for Efficient Use of Water and Nitrogen. ASA, Special Publication No. 15, Wisconsin. pp. 101-113 
Rahman, M. M., Sofian-Azirun, M., \& Boyce, A. N. (2013). Response of nitrogen fertilizer and legumes residues on biomass production and utilization in rice-legumes rotation. The Journal of Animal \& Plant Sciences, 23(2), 589-595.

Raun, W. R., \& Johnson, G. V. (1999). Improving nitrogen use efficiency for cereal production. Agronomy Journal, 91, 357-363. https://doi.org/10.2134/agronj1999.00021962009100030001x

Sage, R. F., \& Zhu, X. G. (2011). Exploiting the engine of C4 photosynthesis. Journal of experimental botany, 62(9), 2989-3000.

Sebetha, E. (2015). The effect of maize-legume cropping system and nitrogen fertilization on yield, soil organic carbon and soil moisture. (Master's thesis, University of KwaZulu-Nata, South Africal ).

Sowers, K. E., Pan, W. L., Miller, B. C., \& Smith, J. L. (1994). Nitrogen use efficiency of split nitrogen applications in soft white winter wheat. Agronomy Journal, 86, 942-948. https://doi.org/10.2134/agronj1994.00021962008600060004x

Walworth, J. (2013). Nitrogen in soil and the environment. University of Arizona, college of Agriculture and life sciences. Retrieved from www.cals.Arizona.edu/pubs. Accessed on 13/03/2016.

Werner, D., \& Newton, W. E. (2005). Nitrogen fixation in agriculture, forestry, ecology, and the environment. Springer Publication. https://doi.org/10.1007/1-4020-3544-6

Westermann, D. T., Kleinkopf, G. E., Porter, L. K., \& Leggett, G. E. (1981). Nitrogen sources for bean seed production. Agronomy Journal, 73, 660-664. https://doi.org/10.2134/agronj1981.00021962007300040022x

Zhao, R. F., Chen, X. P., Zhang, F. S., Zhang, H., Schroder, J., \& Romheld, V. (2006). Fertilization and N balance in a wheat-maize rotation system in North China. Agronomy Journal, 98(4), 938-945. https://doi.org/10.2134/agronj2005.0157

\section{Copyrights}

Copyright for this article is retained by the author(s), with first publication rights granted to the journal.

This is an open-access article distributed under the terms and conditions of the Creative Commons Attribution license (http://creativecommons.org/licenses/by/3.0/). 